\title{
Una aproximación a lo que son las necesidades de información
}

\author{
Juan José Calva González"
}

\section{RESUMEN}

Se expone cómo surgen las necesidades humanas, así como algunas de sus clasificaciones. Asimismo, analiza cómo se organizan las necesidades de información en el ser humano; y por último se explica cuál es la disciplina encargada de satisfacerlas.

Expone a la Bibliotecología como la disciplina que tiene como objeto de estudio la información y que además es la encargada de satisfacer las necesidades de información de los usuarios que acuden a la biblioteca. Se considera que la biblioteca es una institución creada por la sociedad para obtener la información que genera la propia sociedad, además es la institución que conserva, organiza y difunde la información.

\begin{abstract}
A description is made pf haw human needs arise, and also how they can be classified. In a similar manner, this articles analyses how the need for information arises within the human being. Finally, an explanation is provided regarding the academic discipline that is responsible for satisfying that need.

Library Science is then described as the academic discipline that has as its purpose the study of information. Additionally, it is the discipline entrusted with satisfying the information needs of library users. The library is considered an institution created by society in order to obtain information generated by the same society. It is also the institution that preserves, organizes and diffuses that information.
\end{abstract}

\section{INTRODUCCIÓN}

$\boldsymbol{E}$ xiste una relación real entre información y bibliotecología, y se puede considerar que la material prima de la bibliotecología es la información. ${ }^{1}$ Sin embargo parece que esta relación no estaba claramente definida, creyéndose, tal vez, que la relación era exclusivamente con el libro y no con lo que contenía el libro.

Actualmente, dados los avances tecnológicos y la notable influencia que éstos han tenido en todas las actividades humanas, es necesario que se reafirmen muchas disciplinas, entre ellas las bibliotecología, ya que, como se escucha, si el libro alguna vez desaparece, o como ocurre actualmente, empiezan a surgir otros medios como cintas de audio, o video, discos compactos, etcétera, la bibliotecología como disciplina dejaría de existir, para dar paso a otra u otras disciplinas.
Por lo anterior es importante que se reafirme la disciplina bibliotecológica, y amplíe su concepto y por lo tanto su horizonte, que a final de cuentas, siempre será el mismo: la información.

Dado que la disciplina trabaja con la información como materia prima debe de investigarse a fondo el ente que necesita de esta materia prima, que en nuestro planeta es sólo el ser humano y él es el único que presenta necesidades de información.

\section{EL SER HUMANO}

El hombre es producto de una larga evolución; es un organismo altamente desarrollado. El cerebro humano es un órgano complejo, que ofrece muchas oportunidades de establecer nuevos modelos de conducta y nuevas pautas culturales en periodos cortos de tiempo. ${ }^{2}$

El hombre desde el punto de vista de la clasificación de los organismos pertenece al:

REINO ANIMAL ${ }^{3}$

PHYLUM: CHORDATA

CLASE: MAMÍFERO

ORDEN: PRIMATES

FAMILIA: HOMÍNIDO

GÉNERO: HOMO

ESPECIE: SAPIENS (ÚNICA)

El ser humano, a diferencia de otros animales, tiene una dependencia del sujeto adulto cuando es pequeño y posee gran capacidad para adaptarse al entorno físico y cultural. ${ }^{4}$ Así el difícil proceso de maduración del niño viene compensado 
por su capacidad de aprendizaje, lo cual incrementa, de gran manera, las posibilidades de supervivencia de la especie humana. 5

"Casi todos los organismos terrestres actúan en buena medida conforme al legado genético de que son portadores y que ha sido previamente transmitido al sistema nervioso del individuo, siendo la información extragenética recogida en el curso de su vida un factor secundario". 6 En el caso del hombre ocurre que la información extragenética recogida en el transcurso de la vida es de vital importancia sin desconocer el notable influjo del legado genético en el comportamiento del hombre.

El cerebro humano durante su larga evolución ha procurado obtener información acumulada fuera del cuerpo, de la cual la escritura constituye el ejemplo más significativo, lo que representa conocimiento extragenético. ${ }^{7}$

La capacidad de aprendizaje del ser humano le ha permitido ajustarse a adaptarse al cambio y controlarlo. El mecanismo de aprendizaje ha permitido el desarrollo y transmisión de la información acumulada a través de la escritura; por lo anterior es posible afrontar el rapidísimo proceso de transformación que soporta la especie humana. ${ }^{8}$

El ser humano puede ser considerado como un procesador de información; ${ }^{9}$ a medida que ésta entra a través de los órganos de los sentidos es procesada por el sistema nervioso, de hecho primeramente la información verbal y escrita dotada de significado acústico y visual es la que es percibida por los sentidos y procesada por el sistema nervioso; éste introduce alteraciones son simplificaciones que sólo contienen la información sustancial y llegan simplificadas a niveles más altos; así la información que entra en el sistema nervioso es procesada y una vez en el cerebro puede generar conocimiento. ${ }^{10}$

El conocimiento generado por el cerebro produce nueva información que para transmitirla y conservarla fuera del cuerpo utiliza la escritura para que ésta, a su vez, pueda ser captada a través de los sentidos por otro ser humano y pueda en un momento dado generar nuevo conocimiento. Así, la escritura, como gran invención del hombre para comunicarse, tiene un origen oscuro a millones de años en el paleolítico. ${ }^{11}$

La información procesada y el conocimiento generado permite al hombre adaptarse a los cambios del ambiente para sobrevivir.

Indudablemente el sistema nervioso humano y su órgano regulador, el cerebro, a través de su larga evolución ha desarrollado la capacidad de no sólo procesar la información que percibe, sino además almacenarla fuera del propio cerebro, esto lo ha logrado utilizando símbolos, figuras y sonidos. Como señales de esto se pueden citar las pinturas rupestres, construcciones monolíticas, los jeroglíficos, papiros, tablillas de arcilla, manuscritos, libros, material audiovisual y, actualmente, medios electrónicos (computadoras) y discos compactos.

A través del sistema nervioso los organismos deben ser capaces de responder a cambios físicos y químicos del medio ambiente para poder sobrevivir. Los cambios en el medio ambiente son lo que causan una respuesta, un cambio o una reacción en el organismo. De esta forma el sistema nervioso es el productor de algo que poseen los seres humanos que son las emociones.

Asimismo el sistema nervioso y sus intrincados mecanismos nerviosos, están sintonizados para percibir alteraciones del medio ambiente externo e interno, ya que oye, ve, siente, analiza y considera, realiza y reacciona a ellos. ${ }^{12}$

\section{LAS NECESIDADES HUMANAS}

Las necesidades humanas son manifestaciones de reacciones que tiene el individuo, ya sea por causas básicas o de más alto nivel (que es cuando intervienen factores ambientales externos al organismo); todas estas reacciones son manifestadas a través del sistema nervioso.

Para comprender las necesidades humanas se ha tratado de ponerlas por niveles de importancia o jerarquía, pero indudablemente si tomamos en cuenta el desarrollo humano, ya sea desde el punto de vista como especie o como individuo (etapas de crecimiento), las necesidades se presentan como una gran gama, pero todas ellas relacionadas con el sistema nervioso, puesto que se manifiestan a través de conductas que éste regula y controla en el individuo.

Las necesidades pueden agruparse según su importancia y de acuerdo a como se van presentando; de esta forma existen las necesidades básicas o fisiológicas que presentan todos los organismos vivos; y después surgen otras necesidades del nivel más alto que dependiendo del sujeto se vuelven más complejas.

Las necesidades básicas o fisiológicas son aquellas como la sed, el hambre, etcétera y son las que mueven, estimulan o motivan al organismo más ávidamente para satisfacerlas; ninguna necesidad de más alto nivel puede surgir mientras éstas no estén relativamente satisfechas. De esta forma mientras el organismo tenga hambre no estará en estabilidad hasta que no haya satisfecho esta necesidad.

Se han tratado de ordenar, nivelar o jerarquizar las necesidades humanas. Henry Murray ${ }^{13}$ identifica 20 necesidades humanas, que se mencionan a continuación:

\section{- Necesidad de abatimiento o} degradación

- Necesidad de realización

- Necesidad de afiliación

- Necesidad de agresión

- Necesidad de autonomía

- Necesidad de oposición

- Necesidad de defensa

- Necesidad de diferenciación

- Necesidad de dominación

- Necesidad de exhibición

- Necesidad de evitar perjudicar o dañar

- Necesidad de prevención

- Necesidad de promoción

- Necesidad de orden

- Necesidad de juego

- Necesidad de rechazo

- Necesidad de sensibilidad o percepción

- Necesidad de sexo

- Necesidad de ayudar o socorrer

- Necesidad de entendimiento o comprensión (en esta última se podría situar la necesidad de información).

Las 20 necesidades humanas que encontró Murria las agrupa en lo que llamó necesidades psicogenéticas, y existen otras más llamadas necesidades vicerogenéticas donde se incluyen las necesidades de 
comida, agua y oxígeno, ${ }^{14}$ en estas últimas se sitúan las necesidades básicas o fisiológicas mencionadas anteriormente.

Existen otras proposiciones que jerarquizan las necesidades humanas, es el caso de Abraham Maslow quien realizó una jerarquía de necesidades humanas de la siguiente forma:

- Necesidades fisológicas

- Necesidades de seguridad

- Necesidades sociales

- Necesidades de estima

- Necesidades de autorrealización

Al ser humano una vez que va satisfaciendo cada nivel de necesidades le surgen otras del nivel siguiente superior, así hasta tender al nivel más alto que es el de autorrealización. ${ }^{15}$

El nivel más alto es aquel en el cual el individuo puede desarrollar todo su potencial físico e intelectual para llevar a cabo sus actividades. ${ }^{16}$

Hay que considerar que en este último nivel se ubican las necesidades de conocimiento o entendimiento, puesto que, si el ser humano quiere desarrollar todo su potencial deberá conocer las cosas que lo rodean y que tienen vinculación con la actividad que desempeña. ${ }^{17}$

Sin embargo, el hombre tiende siempre hacia el nivel de autorrealización, mas no permanece estable en él, ya que siempre habrá algo nuevo por perfeccionar o un conocimiento nuevo por adquirir. Además siempre resurgen las necesidades de los otros niveles inferiores que anteriormente ya estaban satisfechas.

Si el sujeto se encuentra en o tiende al nivel de autorrealización, puede ser posible que genere conocimiento nuevo sobre su campo de trabajo siempre y cuando reciba información, la cual procesa en el cerebro y puede llegar a generar a su vez conocimiento; pudiera darse que, por no encontrarse en este nivel, sólo recibe información pero no genera nuevo conocimiento y por lo tanto nueva información.

\section{LAS NECESIDADES DE INFORMACIÓN}

Dentro de las necesidades humanas en su más alto nivel aparecen las necesidades de información que surgen en las personas cuando se encuentran en una situación en la cual requieren determinado conocimiento. Es entonces cuando el sujeto recibe un estímulo el cual da como resultado la necesidad de información, la que el sujeto trata de satisfacer. De esta forma las necesidades de información son el resultado de los problemas que se le presentan a un individuo en una situación específica. $^{18}$

El cerebro, como órgano, permite en gran medida la superviencia de las personas, a través de dar pautas para adaptarse al medio ambiente, pero para esto necesita tener la información del medio cambiante. ${ }^{19}$ Así, el cerebro necesita procesar la información recibida y dar una respuesta.

La necesidad del cerebro de procesar y recibir constantemente nueva información es una característica inherente de los animales superiores; una pequeña porción del sistema nervioso es desarrollado al nacer, pero la mayor parte se desarrolla continuamente a través de la estimulación y la experiencia, que se da por medio de la absorción, organización y procesamiento de toda la información obtenida por medio del sentido de la percepción. $^{20}$

El medio ambiente, en el cual se encuentra el ser humano, está plagado de información escrita, sonora y visual. El ser humano debe procesar esta información, ya sea para producir nuevo conocimiento (y adaptarse al medio ambiente) o para generar más información, o simplemente por tenerla solo para sí.

¿La necesidad de información se sitúa en el nivel más alto de la jerarquía de necesidades humanas? Para dar respuesta tentativa a esta incógnita, hay que considerar que el individuo ya debe haber cubierto sus necesidades básicas y que se encuentra influenciado por factores externos (medio ambiente), los cuales lo estimularán a que tenga una reacción; deberá adaptarse a él, para lo cual necesita que el cerebro, al procesar la información recibida, emita una respuesta generando

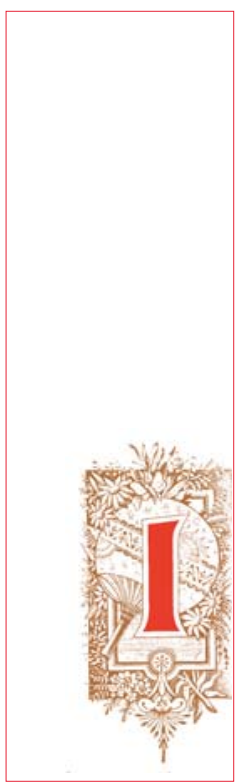

\author{
Dentro de las necesidades \\ humanas en su más alto nivel \\ aparecen las necesidades de \\ información que surgen en \\ las personas cuando se \\ encuentran en una situación \\ en la cual requieren \\ determinado conocimiento
}

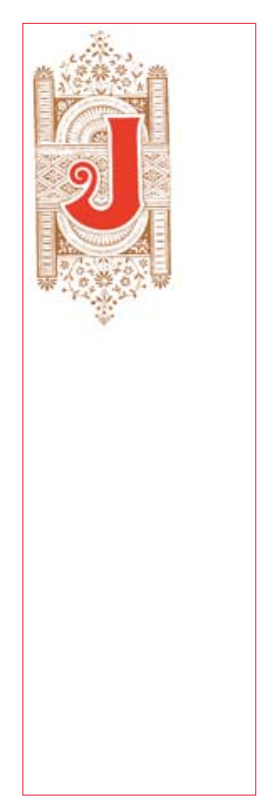


conocimiento que le permita al organismo adaptarse al medio.

Es indudable que el hombre necesita de la información extragenética acumulada fuera de su cuerpo para poder utilizarla en su adaptación al medio ambiente.

La construcción del término necesidades de información es una construcción abstracta usada para responder por qué las personas buscan, encuentran y usan la información. ${ }^{21}$ Usualmente se le da diferentes interpretaciones, así como se dan confusiones con otros términos como: demandas de información, requerimientos de información o deseos de información. ${ }^{22}$ Pero hay que considerar que el término necesidad tiene una connotación muy profunda (como se ha visto) e información está definida como: conocimientos, ideas, hechos, datos o trabajos creativos o imaginativos producidos por la mente, los cuales son comunicados formalmente y/o informalmente en cualquier formato. $^{23}$

Asimismo hay que considerar que la búsqueda, requerimiento, demanda o deseo de información, es el reflejo de que existe una necesidad de información en la persona

Si el individuo presenta una necesidad de información es porque la necesita en ese momento, puesto que se ha visto estimulado, a través de su sistema nervioso, a satisfacerla.

Hay dos caminos para satisfacer la necesidad de información: buscar los conocimientos acumulados por hechos, podría entenderse por el mismo proceso de aprendizaje (experiencia); y aprovechar la información que produce uno mismo, es decir, la que es producto de la actividad humana ${ }^{24}$ y la cual se encuentra registrada.

Las necesidades de información se presentan en todos los aspectos de la vida diaria en el hogar, en la oficina, en las relaciones familiares y con las amistades. También se preenta por curiosidad o por requerimientos de trabajo. ${ }^{25} \mathrm{Sin}$ embargo, no sólo las personas presentan una necesidad de información sino también las organizaciones o instituciones (compañías, industrias, fábricas, etc.)pero sin olvidar que dichas entidades están compuestas por personas. Asimismo personas o entidades pueden ser proveedores de la información al generar conocimientos, ideas o hechos.

\section{LA BIBLIOTECOLOGÍA COMO DISCIPLINA QUE ESTUDIA CÓMO SATISFACER LAS NECESIDADES INFORMACIÓN. \\ DE}

Mucho se menciona que el fin último de la biblioteca es el usuario y que hay que satisfacer las necesidades de información. Para cumplir con esta tarea es necesario conocer la naturaleza de las necesidades de información, como prioridad sobre la búsqueda y uso de la información.

Primeramente hay que considerar que la biblioteca, en su concepto más amplio, es el lugar creado por el ser humano, para preservar, organizar y diseminar la información, sin importar el tipo de material en que se encuentre la información, ya sea en libros, discos, citas de audio, películas, disco compacto, cintas magnéticas, microfichas o cualquier otro elemento. ${ }^{26}$

El surgimiento de la bibliotecología como disciplina que permita la información de profesionales que conozcan, manejen y optimicen todos los procesos relacionados con el uso de la información, independientemente de donde se encuentren registrados 27 deberá de permitir al hombre satisfacer sus necesidades de información.

La biblioteca es el organismo del cual la sociedad espera obtener información. Sin embargo, es preciso que una disciplina se encargue de entender las necesidades de información de la sociedad, pero no sólo de entenderlas (estudiarlas) sino buscar mecanismos para satisfacerlas plenamente.

El usuario de la biblioteca que ingresa a pedir información sobre algún asunto se encuentra en un nivel de necesidad alto, el cual debe ser satisfecho y acude al lugar adecuado donde puede satisfacerlo.

En la disciplina bibliotecológica se debe estudiar perfectamente las necesidades de información que tiene el usuario para poder satisfacerlas. Como se mencionó en párrafos anteriores la necesidad de información del hombre se presenta en su vida diaria. Asimismo, el hombre como especie necesita información para sobrevivir, lo cual hace a la bibliotecología una disciplina de gran importancia.

Si se conocen las necesidades de información del usuario, al cual sirve la biblioteca, se le estará otorgando un beneficio $\mathrm{y}$, como fin último, también a la sociedad en la cual se encuentra inmerso, ya que esta necesidad del usuario al ser satisfecha puede llegar a generar conocimiento (pero no necesariamente) y por tanto producir más información.

La bibliotecología deberá de formar profesionales que conozcan las necesidades de información, la bibliotecología debe proporcionar los conocimientos de la materia prima con la cual trabaja: la información. De esta forma los profesionales deberían "estudiar el comportamiento de los elementos que en ello intervienen como son la información, los usuarios, métodos de almacenamiento, la recuperación y difusión entre otros". 28

Es imperioso que en la propia disciplina bibliotecológica se realicen investigaciones sobre las necesidades de información, que sustenten la razón de ser de la propia disciplina y de la biblioteca como institución para llegar a último término al usuario.

Podría decirse que el propósito del estudio del bibliotecario es el hombre (el sistema de comunicación neuropsicológico), es decir, los caminos por los cuales el hombre aprende, su lenguaje (primordialmente el escrito) y principalmente su reacción ante el registro de la información y la influencia de los registros de información sobre su conducta, ${ }^{29}$ en suma, estudiar las necesidades de información para poder satisfacerlas. 


\section{NOTAS BIBLIOGRAFICAS}

1. MORALES, Estela. "Bibliotecología e Información". - Boletín de la Asociación Andaluza de Bibliotecarios. - Año 5m bi, 15 jun. 1989. p. 13-21.

2. SAGAN, Carl. Los dragones del edén: especulaciones sobre la evolución de la inteligencia humana: - México: Grijalbo, 1984. - p. 16.

3. Ciencias biológicas: de las moléculas al hombre/ adaptado de la versión azul del Biological Science Currículo Study; Claude A. Wolch... [et. al.]. - México : CECSA, c1989. - p. 79.

4. SAGAN, op. cit,. p. 15.

5. Idem., p. 16.

6. Idem, p. 16.

7. Idem, p. 16.

8. Idem, p. 17.

9. NORMANDAD, D.A. El procesamiento de la información en el hombre.Buenos Aires : Piados, 1972. - p. 16.

10. Idem, p. 17.

11. SHERA, Jesé. Thefoundations of librarianship. - New York : Wiley-Bcker, 1972. - p. 17

12. RANSON, S.W. Anatomía del sistema nervioso. - México : Interamericana, c1963. - p. 1

13. Citado por Henry Murria, en BOURNE, Lyle E. Jr., Bruce R. Ekstrand. Psychology: its principles and meaning. $-2 \mathrm{n}$ ed. $-\tilde{\mathrm{n}}$ - New York : Holt, Rinehart and Winston, c1976. - p. 208

14 Idem. P. 207.

15. MASLOW, A. H. Motivation and personality. - 2nd ed. - New York : Harper and Row, c1970. - p. 38.

16. Idem. P. 46.

17. CHIH-CHIH CHEN. Information seeking. - New York : Neal Schuman, 1987. - p. 5.

18. DURRANCE, J. "Information need". - en Rethinking the library in information age. V. II U. S. Office of Educational Research provement, office of library process, 1988. - p. 159.

19. FRANTS, Valery. "The need for information and some aspects of information retrieval systems constructions". - en Journal of American society for information science. - vol. 39 no. 2, mar. 1988. - p. 86.

20. SHERA, op. cit., p. 8. 
21. CHING, op. cit., p. 8.

22. FAIBISOFF, Sylvia G. and Donald R. Ely "information and information need". - en Information and bibliographies, - vol. 5 no. 5, 1976. - n. 3.

23. CHING-CHIH, op. cit., p. 5.

24. FRANTS, op. cit. p. 82

25. CHING, op. cit., p. 5.

26. MORALES, Estela, op. cit., pp. 13-21.

27. Idem, pp. 13-21.

28. Idem, pp. 13-21.

27. SHERA, op. cit., p. 48.

\section{OBRAS CONSULTADAS}

BOURNE, Lyle E. Jr. Psychology : its principles and meaning. $-2^{\text {nd }}$ ed. New York : Hol, Rinchart and Winston, c1976.

Ciencias biológicas : de las moléculas al hombre / adaptado de la versión azul del Biological Science Currículo Study; Calude A. Wolch... [et. al.]. - México : CECSA, c1978.

CHING-CHIH CHEN, Information seeking. - New York : Neal Schuman, 1982.

DURRANCE, J. "Information need", — en Rethinking the library information age. V. II, U. S. Office of Educational Research provement, office of library process, 1988.

FAIBISOFF, S. "Information and information need". - en Information reports and bibliographies. - vol. 5, no. 5, 1976.

FRANTS, Valery. "The need for information and some aspects of information retrieval systems construction". .. en Journal of American society for information science. - vol. 39, no. 2, mar. 1988.

MASLOW, a. H. Motivation and personality. $-2^{\text {nd }}$. ed. - New York : Harper and Row, c1970.

MORALES, Estela. "Bibliotecología e información". - en Boletín de la Asociación de Bibliotecarios. - Año 5, no. 15, jun. 1989. - pp. 13-21.

NORMAN, D.A. El procesamiento de la información en el hombre. - Buenos Aires, Piados, 1972.

RANSON, S. W. Anatomía del sistema nervioso. - México : Interamericana, c1963.

SAGAN, Carl. Los dragones del edén : especulaciones sobre la evolución de la inteligencia humana. - México : Grijalbo, 1984.

SHERA, Jesé. The foundation of libraianship. — New York: Wiley-Becker, 1972. 